\title{
REGIONAL CONTROL PROBLEM FOR DISTRIBUTED BILINEAR SYSTEMS: APPROACH AND SIMULATIONS
}

\author{
KARIMA ZTOT * , El HASSAN ZERRIK* ${ }^{*}$ HAMID BOURRAY ** \\ * MACS Team, Faculty of Sciences \\ Moulay Ismail University, BP 4010, Béni M’hamed, Zitoune, Meknes, Morocco \\ e-mail: k.ztot@menara.ma; zerrik3@yahoo.fr \\ ** TICOS Team, Faculty of Multidisciplinary Research \\ Moulay Ismail University, BP 512, Boutalamine, 52000, Errachidia, Morocco \\ e-mail: hbourrayh@yahoo.fr
}

\begin{abstract}
This paper investigates the regional control problem for infinite dimensional bilinear systems. We develop an approach that characterizes the optimal control and leads to a numerical algorithm. The obtained results are successfully illustrated by simulations.
\end{abstract}

Keywords: distributed systems, bilinear systems, regional controllability, regional optimal control problem.

\section{Introduction}

The aim of several control problems is to drive a dynamical system from an initial state to a desired one in a finite time. Let us consider a distributed bilinear systems evolving on $\Omega \subset \mathbb{R}^{n}$ and described by the equation

$$
\left\{\begin{array}{l}
\dot{z}(t)=A z(t)+u(t) B z(t), \quad t \in[0, T], \\
z(0)=z_{0} \neq 0,
\end{array}\right.
$$

where $A$ is the generator of a strongly continuous semigroup $(S(t))_{t \geq 0}$ on the state space $Z=: L^{2}(\Omega)$ endowed with its natural inner product $\langle\cdot, \cdot\rangle$, and the corresponding norm $\|\cdot\|, B: Z \rightarrow Z$ is a linear bounded operator, while $u \in L^{2}[0, T]$ is a control. The main result on the controllability of the system (1) is due to the pioneering work by Ball et al. (1982), which shows that, under the above-mentioned conditions, a mild solution $z_{u}$ of (1) associated with the control $u$ exists and the set of reachable states from an initial state $z_{0}$ is of dense complement in the state space. This makes exact controllability difficult to be achieved.

Most results are established for particular bilinear systems (Ball et al., 1982; Joshi, 2005; Lenhart and Liang, 2000; Khapalov, 2002a; 2002b). Later the concept of regional controllability for linear distributed systems has been introduced and developed by El Jai and Zerrik and concerns the transfer of such a system to a desired state only on a region of the system spacial domain. The system (1) is said to be exactly (respectively, approximately) controllable in $\omega \subset \Omega$ if for all $z_{d} \in L^{2}(\omega)$ there exists a control $u \in L^{2}[0, T]$ such that $\chi_{\omega} z_{u}(T)=z_{d}$ (respectively, $\left\|\chi_{\omega} z_{u}(T)-z_{d}\right\|_{L^{2}(\omega)} \leq \varepsilon, \varepsilon>0$ ), where $z_{d}$ is a desired state in the space $L^{2}(\omega), \chi_{\omega}: Z \longrightarrow L^{2}(\omega)$ is the restriction operator to $\omega$. Many results for linear and semi linear systems have been developed (see El Jai et al., 1995; Zerrik and Kamal, 2007; Zerrik et al., 2007).

This concept finds its applications in many real world problems. For example, the physical problem which concerns a tunnel furnace where one has to maintain a prescribed temperature only in a subregion of the furnace. Also there exist systems which are controllable on some subregion $\omega \subset \Omega$ but not controllable in the whole domain $\Omega$ and that controlling regionally a system is cheaper than controlling it in the whole domain (see El Jai et al., 1995). In this paper we discuss an extension of previous works (El Jai et al., 1995; Zerrik and Kamal, 2007; Zerrik et $a l ., 2007)$ on regional controllability for linear and semi linear systems to a bilinear one. More precisely, for the system (1) defined on a spatial domain $\Omega$, a nonempty subset $\omega \subset \Omega$, with a positive Lebesgue measure and a desired state $z_{d}$ in $L^{2}(\omega)$, the problem of regional controllability for (1) consists in finding a control function with minimum energy in an appropriate control space that 
steers (1) from $z_{0}$ to a final state close to $z_{d}$ on $\omega$ at time $T$. This problem may be stated as follows:

$$
\left\{\begin{array}{l}
\text { Find } u \in L^{2}[0, T] \text { which minimizes }\|u\|_{L^{2}[0, T]}^{2}, \\
u \in U_{\mathrm{ad}}(\omega)
\end{array}\right.
$$

while

$$
\begin{aligned}
& U_{\mathrm{ad}}(\omega) \\
& =\left\{u \in L^{2}[0, T]:\left\|\chi_{\omega} z_{u}(T)-z_{d}\right\|_{L^{2}(\omega)} \text { is minimum }\right\} .
\end{aligned}
$$

We discuss the cases of $U_{\text {ad }}(\omega) \neq \emptyset$ and $U_{\text {ad }}(\omega)=\emptyset$. To characterize the optimal solution of (2), we propose an approach based on a quadratic cost control problem, which involves the minimization of the control norm and the final state error. This is the aim of this paper, which is organized as follows. In Section 2 we consider the quadratic cost control problem associated with (2). In Section 3, we give a characterization of a control solution of (2) and we show that, under supplementary conditions, the uniqueness may be ensured. In the last section, we develop a numerical approach and give illustrations with numerical examples and simulations.

\section{Regional quadratic control problem}

Given $T>0$, let us associate with (2) the problem

$$
\min _{u \in L^{2}[0, T]} J_{\epsilon}(u)
$$

with

$$
\begin{aligned}
J_{\epsilon}(u)= & \left\|\chi_{\omega} z_{u}(T)-z_{d}\right\|_{L^{2}(\omega)}^{2} \\
& +\epsilon \int_{0}^{T} u^{2}(t) \mathrm{d} t, \quad \epsilon>0 .
\end{aligned}
$$

\section{Proposition 1.}

1. For $u \in L^{2}[0, T]$ and $h \in L^{2}[0, T], \forall t \in[0, T]$, we have $\left\|z_{u+h}(t)-z_{u}(t)\right\|=o(\|h\|)$ as $h \rightarrow 0$.

2. There exists $u^{*} \in L^{2}[0, T]$ such that

$$
J_{\varepsilon}\left(u^{*}\right)=J^{*}=\min _{v \in L^{2}[0, T]} J_{\varepsilon}(v) .
$$

Let

$$
y(t)=\int_{0}^{t} U(t, s) h(s) B z_{u}(s) \mathrm{d} s
$$

Then

$$
\left\|z_{u+h}(t)-z_{u}(t)-y(t)\right\|=o(\|h\|)
$$

as $h \rightarrow 0$, where $(U(t, s))_{t \geq s}$ is the evolution operator generated by $A+u B$.
Proof.

1. We have

$$
\begin{aligned}
& z_{u+h}(t)-z_{u}(t) \\
& =\int_{0}^{t} S(t-s) u(s) B\left(z_{u+h}(s)-z_{u}(s)\right) \mathrm{d} s \\
& \quad+\int_{0}^{t} S(t-s) h(s) B z_{u+h}(s) \mathrm{d} s .
\end{aligned}
$$

Using the boundedness of the semigroup $(S(t))_{t \geq 0}$ on the entire finite interval of $[0, T]$, i.e., the fact that there is an $M>0$ such that $\|S(t)\| \leq M, \forall t \in[0, T]$, we have

$$
\begin{aligned}
\left\|z_{u+h}(t)-z_{u}(t)\right\| & \\
\leq & M\|B\|\left(\int _ { 0 } ^ { t } \left[|u(s)|\left\|z_{u+h}(s)-z_{u}(s)\right\|\right.\right. \\
\quad & \left.\left.+|h(s)|\left\|z_{u+h}(s)\right\|\right] \mathrm{d} s\right),
\end{aligned}
$$

and

$$
\begin{aligned}
\left\|z_{u+h}(t)\right\| \leq & M\left(\left\|z_{0}\right\|\right. \\
& \left.+\|B\| \int_{0}^{t}|u(s)+h(s)|\left\|z_{u+h}(s)\right\| \mathrm{d} s\right) .
\end{aligned}
$$

Using the Gronwall inequality twice, we obtain $\left\|z_{u+h}(t)\right\| \leq k_{1}$, and

$$
\begin{aligned}
\left\|z_{u+h}(t)-z_{u}(t)\right\| & \\
\leq & M\|B\|\left(\int_{0}^{t}|u(s)|\left\|z_{u+h}(s)-z_{u}(s)\right\| \mathrm{d} s\right. \\
& \left.+k_{1} \int_{0}^{t}|h(s)| \mathrm{d} s\right),
\end{aligned}
$$

and, again by the Gronwall inequality, we obtain $\left\|z_{u+h}(t)-z_{u}(t)\right\|=o(\|h\|)$ as $h \rightarrow 0$.

2. The set $E=\left\{J_{\varepsilon}(u) \mid u \in L^{2}[0, T]\right\}$ is nonempty and bounded from below, so the lower bound $J^{*}$ exists. Let the sequence $\left(u_{n}\right)$ in $L^{2}[0, T]$ be such that

$$
\lim _{n \rightarrow+\infty} J_{\varepsilon}\left(u_{n}\right)=J^{*} .
$$

We have

$$
\varepsilon \int_{0}^{T} u_{n}^{2}(t) \mathrm{d} t \leq J_{\varepsilon}\left(u_{n}\right) .
$$

Hence $\left(u_{n}\right)_{n \geq 0}$ is bounded. Therefore, we can extract a subsequence denoted by $\left(u_{n_{k}}\right)$ which converges weakly to $u^{*}$ in $L^{2}[0, T]$. This implies that $z_{u_{n_{k}}}$ converges to $z_{u^{*}}$ strongly in $\mathcal{C}(0, T ; Z)$ (see Ball et al., 1982). Hence

$$
J_{\varepsilon}\left(u^{*}\right) \leq \liminf _{n \rightarrow \infty} J_{\varepsilon}\left(u_{n}\right)=J^{*} \leq J_{\varepsilon}\left(u^{*}\right) .
$$

3. Since $u \in L^{2}[0, T]$ and $B$ is a bounded linear operator on $Z$, the operator $A+u B \in L^{1}[0, T ; D(A)]$. Then 
$A+u(t) B$ generates an evolution operator $(U(t, s))_{t \geq s}$ (cf. Pazy, 1983, Chapter 5, Remark 3.2). Thus

$$
y(t)=\int_{0}^{t} U(t, s) h(s) B z_{u}(s) \mathrm{d} s
$$

is well defined.

Let $Y(t)=z_{u+h}(t)-z_{u}(t)-y(t)$. We can write

$$
\begin{aligned}
Y(t)= & \int_{0}^{t} S(t-s) u(s) B Y(s) \mathrm{d} s \\
& +\int_{0}^{t} S(t-s) h(s) B\left(z_{u+h}(s)-z_{u}(s)\right) \mathrm{d} s \\
& +\int_{0}^{t} S(t-s) h(s) B z_{u}(s) \mathrm{d} s \\
& +\int_{0}^{t} S(t-s) u(s) B y(s) \mathrm{d} s-y(t) .
\end{aligned}
$$

Let

$$
\begin{aligned}
K(t)= & \int_{0}^{t} S(t-s) h(s) B z_{u}(s) \mathrm{d} s \\
& +\int_{0}^{t} S(t-s) u(s) B y(s) \mathrm{d} s-y(t) .
\end{aligned}
$$

Then, for $z_{0} \in D(A)$, we have

$$
\begin{aligned}
\dot{K}(t)= & A \int_{0}^{t} S(t-s) h(s) B z_{u}(s) \mathrm{d} s \\
& +h(t) B z_{u}(t)+A \int_{0}^{t} S(t-s) u(s) B y(s) \mathrm{d} s \\
& +u(t) B y(t)-\dot{y}(t) .
\end{aligned}
$$

Since $\dot{y}(t)=(A+u(t) B) y(t)+h(t) B z_{u}(t)$ and $y(0)=0$, we get

$$
\begin{aligned}
y(t)= & \int_{0}^{t} S(t-s) h(s) B z_{u}(s) \mathrm{d} s \\
& +\int_{0}^{t} S(t-s) u(s) B y(s) \mathrm{d} s,
\end{aligned}
$$

which shows that $\dot{K}(t)=0$, and since $K(0)=0$, it follows that $K(t)=0, \forall t \in[0, T]$.

Then we have

$$
\begin{aligned}
Y(t)= & \int_{0}^{t} S(t-s) u(s) B Y(s) \mathrm{d} s \\
& +\int_{0}^{t} S(t-s) h(s) B\left(z_{u+h}(s)-z_{u}(s)\right) \mathrm{d} s,
\end{aligned}
$$

and

$$
\begin{aligned}
\|Y(t)\| \leq & M\|B\|\left(\int_{0}^{t}|u(s)|\|Y(s)\| \mathrm{d} s\right. \\
& \left.+\int_{0}^{t}|h(s)|\left\|z_{u+h}(s)-z_{u}(s)\right\| \mathrm{d} s\right) .
\end{aligned}
$$

By Property 1, we have

$$
\begin{aligned}
& M\|B\| \int_{0}^{t}|h(t)|\left\|z_{u+h}(s)-z_{u}(s)\right\| \mathrm{d} s \\
& \leq k_{1}\|h\|^{2}, \quad k_{1} \in \mathbb{R} .
\end{aligned}
$$

By the Gronwall inequality, we obtain

$$
\|Y(t)\| \leq k_{2}\|h\|^{2}, \quad k_{2} \in \mathbb{R}
$$

that is,

$$
\|Y(t)\|=o(\|h\|),
$$

and by the density of $D(A)$ in $Z$ we have the above inequality in $Z$.

Now, the solution to the problem (3) is characterized by the following result.

Theorem 1. A control which minimizes the problem (3) is given by

$$
u(t)=-\frac{1}{\epsilon}\left\langle B z(t), P(t) z(t)-U^{*}(T, t) \chi_{\omega}^{*} z_{d}\right\rangle,
$$

where $P$ is the selfadjoint and nonnegative operator solution of the following equation:

$$
\left\{\begin{array}{l}
\frac{\mathrm{d}}{\mathrm{d} t}\langle P(t) y, z\rangle+\langle P(t) y,(A+u(t) B) z\rangle \\
+\langle(A+u(t) B) y, P(t) z\rangle=0, \quad \forall y, z \in D(A), \\
P(T)=\chi_{\omega}^{*} \chi_{\omega} .
\end{array}\right.
$$

Here $U^{*}(t, s)$ is the adjoint operator of $U(t, s)$ and $\chi_{\omega}^{*}$ is the adjoint operator of $\chi_{\omega}$.

The minimum is given by

$$
\begin{aligned}
J_{\varepsilon}(u)= & \left\langle P(0) z_{0}, z_{0}\right\rangle+2\left\langle\chi_{\omega} U(T, 0) z_{0}, z_{d}\right\rangle \\
& +\left\|z_{d}\right\|_{L^{2}(\omega)}^{2}+\varepsilon \int_{0}^{T} u^{2}(t) \mathrm{d} t .
\end{aligned}
$$

Proof. Using Property 3 of the previous proposition, we have

$$
z_{u+h}(t)=z_{u}(t)+y(t)+o(h) .
$$

Then we obtain

$$
\begin{aligned}
& \left\langle\chi_{\omega} z_{u+h}(t)-z_{d}, \chi_{\omega} z_{u+h}(t)-z_{d}\right\rangle \\
& =\left\langle\chi_{\omega} z_{u}(t)-z_{d}, \chi_{\omega} z_{u}(t)-z_{d}\right\rangle \\
& \quad+2\left\langle\chi_{\omega} z_{u}(t)-z_{d}, \chi_{\omega} y(t)\right\rangle+o(\|h\|) .
\end{aligned}
$$

Hence

$$
\begin{aligned}
J_{\varepsilon}(u+h)-J_{\varepsilon}(u)= & 2\left\langle\chi_{\omega} z_{u}(T)-z_{d}, \chi_{\omega} y(T)\right\rangle \\
& +2 \varepsilon \int_{0}^{T} u(t) h(t) \mathrm{d} t+o(\|h\|) .
\end{aligned}
$$


For $v \in L^{2}[0, T]$, define

$$
\Lambda v(t)=\chi_{\omega} U(T, t) v(t) B z_{u}(t) .
$$

Since

$$
y(T)=\int_{0}^{T} U(T, s) h(s) B z_{u}(s) \mathrm{d} s,
$$

we obtain

$$
\begin{aligned}
J_{\varepsilon}(u+h)-J_{\varepsilon}(u) \\
=2 \int_{0}^{T}\left[\left\langle\chi_{\omega} z_{u}(T)-z_{d}, \Lambda h(t)\right\rangle \varepsilon u(t) h(t)\right] \mathrm{d} t \\
\quad+o(\|h\|) .
\end{aligned}
$$

Thus, the differential of $J_{\varepsilon}$ at $u$ is

$$
\begin{aligned}
\mathrm{d} J_{\varepsilon}(u) h= & 2 \int_{0}^{T}\left[\left\langle\Lambda^{*}\left(\chi_{\omega} z_{u}(T)-z_{d}\right), h(t)\right\rangle\right. \\
& +\varepsilon u(t) h(t)] \mathrm{d} t .
\end{aligned}
$$

Then $\mathrm{d} J_{\varepsilon}(u) h=0, \forall h \in L^{2}[0, T]$ is equivalent to

$$
u(t)=-\frac{1}{\varepsilon} \Lambda^{*}\left(\chi_{\omega} z_{u}(T)-z_{d}\right) .
$$

Also, we have

$$
\Lambda^{*} v(t)=z_{u}^{*}(t) B^{*} U^{*}(T, t) \chi_{\omega}^{*} v(t),
$$

which gives

$$
u(t)=-\frac{1}{\varepsilon} z_{u}^{*}(t) B^{*} U^{*}(T, t)\left(\chi_{\omega}^{*} \chi_{\omega} z_{u}(T)-\chi_{\omega}^{*} z_{d}\right) .
$$

Let us consider the following nonnegative and selfadjoint operator:

$$
P(t) z=U^{*}(T, t) \chi_{\omega}^{*} \chi_{\omega} U(T, t) z, \quad \forall z \in D(A) .
$$

Since $z_{u}(t)=U(t, 0) z_{0}$, we have

$$
P(t) z_{u}(t)=U^{*}(T, t) \chi_{\omega}^{*} \chi_{\omega} z_{u}(T),
$$

and then we obtain (5).

Let us show that $P(t)$ satisfies Eqn. (6). We have

$$
\frac{\partial U}{\partial s}(t, s) z=-U(t, s)(A+u(s) B) z, \quad \forall z \in D(A) .
$$

Then, $\forall y, z \in D(A)$ and we obtain

$$
\begin{aligned}
\frac{\mathrm{d}}{\mathrm{d} t}\left\langle U^{*}(T, t) \chi_{\omega}^{*} \chi_{\omega} U(T, t) y, z\right\rangle \\
=-\left\langle\chi_{\omega} U(T, t)(A+u(t) B) y, \chi_{\omega} U(T, t) z\right\rangle \\
\quad-\left\langle\chi_{\omega} U(T, t) y, \chi_{\omega} U(T, t)(A+u(t) B) z\right\rangle
\end{aligned}
$$

which shows the right part of (6). Now, using

$$
\begin{gathered}
z_{u}(t)=U(t, 0) z_{0}, \\
\left\langle\chi_{\omega} z_{u}(T), \chi_{\omega} z_{u}(T)\right\rangle=\left\langle P(0) z_{0}, z_{0}\right\rangle,
\end{gathered}
$$

we have

$$
\begin{aligned}
J_{\varepsilon}(u)= & \left\langle P(0) z_{0}, z_{0}\right\rangle+2\left\langle\chi_{\omega} U(T, 0) z_{0}, z_{d}\right\rangle \\
& +\left\|z_{d}\right\|_{L^{2}(\omega)}^{2}+\varepsilon \int_{0}^{T} u^{2}(t) \mathrm{d} t .
\end{aligned}
$$

Remark 1. Equation (6) has a unique solution (cf. El Alami, 1988).

If $u_{\epsilon}$ denotes the solution of (3) and $z_{\epsilon}$ the associated state of (1), the following result will be useful for the sequel of the paper.

\section{Proposition 2.}

1. The sequence $\left(J_{\epsilon}\left(u_{\epsilon}\right)\right)_{\epsilon>0}$ is decreasing as $\epsilon \rightarrow 0$.

2. The sequence

$$
\left(\int_{0}^{T} u_{\epsilon}^{2}(t) \mathrm{d} t\right)_{\epsilon>0}
$$

is increasing as $\epsilon \rightarrow 0$.

3. The sequence $\left(\left\|\chi_{\omega} z_{\epsilon}(T)-z_{d}\right\|_{L^{2}(\omega)}^{2}\right)_{\epsilon>0}$ is decreasing as $\epsilon \rightarrow 0$, and $\forall \epsilon>0$

$$
\left\|\chi_{\omega} z_{\epsilon}(T)-z_{d}\right\|_{L^{2}(\omega)} \leq\left\|\chi_{\omega} S(T) z_{0}-z_{d}\right\|_{L^{2}(\omega)} .
$$

In particular, there exists a subsequence of $\left(\chi_{\omega} z_{\epsilon}(T)-\right.$ $\left.z_{d}\right)_{\epsilon>0}$ which converges weakly in $L^{2}(\omega)$.

Proof. Let $0<\epsilon_{1}<\epsilon_{2}$. Using consecutively the optimality of $u_{\epsilon_{1}}$ for $J_{\epsilon_{1}}$ and the optimality of $u_{\epsilon_{2}}$ for $J_{\epsilon_{2}}$, we have

$$
\begin{aligned}
J_{\epsilon_{1}}\left(u_{\epsilon_{1}}\right) & =\left\|\chi_{\omega} z_{\epsilon_{1}}(T)-z_{d}\right\|_{L^{2}(\omega)}^{2}+\epsilon_{1} \int_{0}^{T} u_{\epsilon_{1}}^{2}(t) \mathrm{d} t \\
& \leq\left\|\chi_{\omega} z_{\epsilon_{2}}(T)-z_{d}\right\|_{L^{2}(\omega)}^{2}+\epsilon_{1} \int_{0}^{T} u_{\epsilon_{2}}^{2}(t) \mathrm{d} t \\
& \leq\left\|\chi_{\omega} z_{\epsilon_{2}}(T)-z_{d}\right\|_{L^{2}(\omega)}^{2}+\epsilon_{2} \int_{0}^{T} u_{\epsilon_{2}}^{2}(t) \mathrm{d} t \\
& \leq\left\|\chi_{\omega} z_{\epsilon_{1}}(T)-z_{d}\right\|_{L^{2}(\omega)}^{2}+\epsilon_{2} \int_{0}^{T} u_{\epsilon_{1}}^{2}(t) \mathrm{d} t .
\end{aligned}
$$

This implies that

$$
J_{\epsilon_{1}}\left(u_{\epsilon_{1}}\right) \leq J_{\epsilon_{2}}\left(u_{\epsilon_{2}}\right) .
$$

From (7), we obtain

$$
J_{\epsilon_{2}}\left(u_{\epsilon_{2}}\right)-J_{\epsilon_{1}}\left(u_{\epsilon_{2}}\right) \leq J_{\epsilon_{2}}\left(u_{\epsilon_{1}}\right)-J_{\epsilon_{1}}\left(u_{\epsilon_{1}}\right),
$$


and then

$$
\int_{0}^{T} u_{\epsilon_{2}}^{2}(t) \mathrm{d} t \leq \int_{0}^{T} u_{\epsilon_{1}}^{2}(t) \mathrm{d} t
$$

Thus $\left\|\chi_{\omega} z_{\epsilon_{1}}(T)-z_{d}\right\|_{L^{2}(\omega)}^{2} \leq\left\|\chi_{\omega} z_{\epsilon_{2}}(T)-z_{d}\right\|_{L^{2}(\omega)}^{2}$, which shows Statements 1 and 2 and the first part of Statement 3.

For $u=0$, we have $z_{u}(T)=S(T) z_{0}$ and $\forall \epsilon>0$,

$$
\begin{aligned}
\left\|\chi_{\omega} z_{\epsilon}(T)-z_{d}\right\|_{L^{2}(\omega)}^{2}+ & \epsilon \int_{0}^{T} u_{\epsilon}(t)^{2} \mathrm{~d} t \\
& \leq\left\|\chi_{\omega} S(T) z_{0}-z_{d}\right\|_{L^{2}(\omega)}^{2} .
\end{aligned}
$$

Then

$$
\begin{aligned}
0 & \leq\left\|\chi_{\omega} z_{\epsilon}(T)-z_{d}\right\|_{L^{2}(\omega)}^{2} \\
& \leq\left\|\chi_{\omega} S(t) z_{0}-z_{d}\right\|_{L^{2}(\omega)}^{2}, \quad \forall \epsilon>0 .
\end{aligned}
$$

Finally, $\left(\left\|\chi_{\omega} z_{\epsilon}(T)-z_{d}\right\|_{L^{2}(\omega)}\right)_{\epsilon>0}$ is bounded. Then we can extract a subsequence of $\left(\chi_{\omega} z_{\epsilon}(T)-z_{d}\right)_{\epsilon>0}$ which converges weakly in $L^{2}(\omega)$.

\section{Regional minimum energy control problem}

Here let us go back to the problem (2), and consider the set

$$
R(T)=\bigcup_{u \in L^{2}[0, T]}\left\{z_{u}(T)\right\}
$$

of the states reachable at time $T$ from $z_{0}$.

We have the main result.

Theorem 2. Let $u_{\epsilon}$ be a solution of (3) and assume that $U_{a d}(\omega)$ is nonempty. Then we have

$$
u_{\epsilon} \rightarrow u^{\star} \quad \text { as } \epsilon \rightarrow 0 \text { in } L^{2}[0, T]
$$

and

$$
\chi_{\omega} z_{\epsilon} \rightarrow \chi_{\omega} z_{u^{\star}} \quad \text { in } C\left([0, T] ; L^{2}(\omega)\right) .
$$

Moreover, $u^{\star}$ is a solution to the problem (2).

Proof. Using the optimality of $u_{\epsilon}$ for $J_{\epsilon}$, we have $\forall \epsilon>0$, $\forall u \in L^{2}[0, T], J_{\epsilon}\left(u_{\epsilon}\right) \leq J_{\epsilon}(u)$, i.e.,

$$
\begin{aligned}
\| \chi_{\omega} z_{\epsilon}(T) & -z_{d} \|_{L^{2}(\omega)}^{2}+\epsilon \int_{0}^{T} u_{\epsilon}^{2}(t) \mathrm{d} t \\
& \leq\left\|\chi_{\omega} z_{u}(T)-z_{d}\right\|_{L^{2}(\omega)}^{2}+\epsilon \int_{0}^{T} u^{2}(t) \mathrm{d} t .
\end{aligned}
$$

$U_{\text {ad }}(\omega)$ is nonempty, which means that there exists $v \in$ $L^{2}[0, T]$ such that

$$
\left\|\chi_{\omega} z_{v}(T)-z_{d}\right\|_{L^{2}(\omega)}^{2}=\min _{z \in R(T)}\left\|\chi_{\omega} z-z_{d}\right\|_{L^{2}(\omega)}^{2} .
$$

Thus, we have

$$
\int_{0}^{T} u_{\epsilon}^{2}(t) \mathrm{d} t \leq \int_{0}^{T} u^{2}(t) \mathrm{d} t, \quad \forall u \in U_{\mathrm{ad}}(\omega), \forall \epsilon>0 .
$$

Therefore, we can extract a subsequence, also denoted by $\left(u_{\epsilon}\right)_{\epsilon>0}$, such that $u_{\epsilon} \rightarrow u^{\star}$ weakly in $L^{2}[0, T]$ and $z_{\epsilon} \rightarrow z_{u^{\star}}$ strongly in $C([0, T] ; Z)$ as $\epsilon \rightarrow 0$ (see Ball et al., 1982), and this implies that $\chi_{\omega} z_{\epsilon} \rightarrow \chi_{\omega} z_{u^{\star}}$ strongly in $C\left([0, T] ; L^{2}(\omega)\right)$. Since $u_{\epsilon} \rightarrow u^{\star}$ weakly in $L^{2}[0, T]$, by the lower semi-continuity of the norm we have

$$
\liminf _{\epsilon \rightarrow 0} \int_{0}^{T} u_{\epsilon}^{2}(t) \mathrm{d} t \geq \int_{0}^{T} u^{\star 2}(t) \mathrm{d} t
$$

and

$$
\liminf _{\epsilon \rightarrow 0} J_{\epsilon}\left(u_{\epsilon}\right) \geq\left\|\chi_{\omega} z_{u^{\star}}(T)-z_{d}\right\|_{L^{2}(\omega)}^{2} .
$$

Moreover, $J_{\epsilon}\left(u_{\epsilon}\right) \leq J_{\epsilon}(u) \forall u \in L^{2}[0, T]$, so

$$
\begin{aligned}
& \limsup _{\epsilon \rightarrow 0} J_{\epsilon}\left(u_{\epsilon}\right) \\
& \quad \leq\left\|\chi_{\omega} z_{u}(T)-z_{d}\right\|_{L^{2}(\omega)}^{2} \quad \forall u \in L^{2}[0, T],
\end{aligned}
$$

and, in particular,

$$
\begin{aligned}
\limsup _{\epsilon \rightarrow 0} J_{\epsilon}\left(u_{\epsilon}\right) & \leq\left\|\chi_{\omega} z_{v}(T)-z_{d}\right\|_{L^{2}(\omega)}^{2} \\
& \leq\left\|\chi_{\omega} z_{u^{\star}}(T)-z_{d}\right\|_{L^{2}(\omega)}^{2} \\
& \leq \liminf _{\epsilon \rightarrow 0} J_{\epsilon}\left(u_{\epsilon}\right) .
\end{aligned}
$$

Hence

$$
\begin{aligned}
\lim _{\epsilon \rightarrow 0} J_{\epsilon}\left(u_{\epsilon}\right) & =\lim _{\epsilon \rightarrow 0}\left\|\chi_{\omega} z_{\epsilon}(T)-z_{d}\right\|_{L^{2}(\omega)}^{2} \\
& =\left\|\chi_{\omega} z_{u^{\star}}(T)-z_{d}\right\|_{L^{2}(\omega)}^{2} \\
& =\left\|\chi_{\omega} z_{v}(T)-z_{d}\right\|_{L^{2}(\omega)}^{2} .
\end{aligned}
$$

Thus

$$
\lim _{\epsilon \rightarrow 0}\left\|\chi_{\omega} z_{\epsilon}(T)-z_{d}\right\|_{L^{2}(\omega)}^{2}=\min _{z \in R(T)}\left\|\chi_{\omega} z-z_{d}\right\|_{L^{2}(\omega)}^{2},
$$

and $u^{\star} \in U_{\text {ad }}(\omega)$.

Furthermore,

$$
\begin{aligned}
& \left\|\chi_{\omega} z_{\epsilon}(T)-z_{d}\right\|_{L^{2}(\omega)}^{2}+\epsilon \int_{0}^{T} u_{\epsilon}^{2}(t) \mathrm{d} t \\
& \quad \leq\left\|\chi_{\omega} z_{u^{\star}}(T)-z_{d}\right\|_{L^{2}(\omega)}^{2}+\epsilon \int_{0}^{T} u^{\star 2}(t) \mathrm{d} t .
\end{aligned}
$$

From (12) it follows that

$$
\int_{0}^{T} u_{\epsilon}^{2}(t) \mathrm{d} t \leq \int_{0}^{T} u^{\star 2}(t) \mathrm{d} t, \quad \forall \epsilon>0 .
$$


Equations (10) and (13) show that

$$
\int_{0}^{T} u_{\epsilon}^{2}(t) \mathrm{d} t \rightarrow \int_{0}^{T} u^{\star 2}(t) \mathrm{d} t \quad \text { as } \epsilon \rightarrow 0 .
$$

This result, together with the weak convergence of $\left(u_{\epsilon}\right)_{\epsilon>0}$ towards $u^{\star}$ in $L^{2}[0, T]$, implies that

$$
\lim _{\epsilon \rightarrow 0} \int_{0}^{T}\left(u_{\epsilon}(t)-u^{\star}(t)\right)^{2} \mathrm{~d} t=0 .
$$

Using (9), we obtain

$$
\int_{0}^{T} u^{\star 2}(t) \mathrm{d} t \leq \int_{0}^{T} u^{2}(t) \mathrm{d} t, \quad \forall u \in U_{\text {ad }}(\omega),
$$

and hence $u^{\star}$ is a solution to the problem (2).

\section{Remark 2.}

1. From the proof of Theorem 2, it follows that, if the sequence $\left(u_{\epsilon}\right)_{\epsilon>0}$ is bounded in $L^{2}[0, T]$, then $U_{\text {ad }}(\omega) \neq \emptyset$.

2 . We do not give any result for the uniqueness except for the global case $(\omega=\Omega)$. We have the following result.

Proposition 3. Suppose that $U_{\mathrm{ad}}(\Omega)$ is nonempty and $L^{2}(\Omega)$ has an orthonormal basis $\left(\phi_{n}\right)_{n}$ of eigenfunctions of $A$. In addition, if $A$ commutes with $B$, then the problem (2) has only one solution.

Proof. First, the existence of a solution is ensured by Theorem 2. With no loss of generality, we may suppose that the eigenvalues of $A$ are simple. Now, $A$ and $B$ commute, so the mild solution of (1) can be written as

$$
z_{u}(t)=S(t) \exp \left(B \int_{0}^{t} u(s) \mathrm{d} s\right) z_{0},
$$

where $\left(\exp \left(B \int_{s}^{t} u(r) \mathrm{d} r\right)\right)_{t>s}$ is the evolution operator generated by $u B$. For $z_{0} \in L^{2}(\Omega)$, we have

$$
z_{u}(t)=\sum_{n=1}^{+\infty} \exp \lambda_{n} t\left\langle\exp \left(B \int_{0}^{t} u(s) \mathrm{d} s\right) z_{0}, \phi_{n}\right\rangle \phi_{n} .
$$

Then

$$
\begin{aligned}
& z_{u}(T)-z_{d} \\
& =\sum_{n=1}^{+\infty}\left\langle\exp \left(\lambda_{n} T\right) \exp \left(B \int_{0}^{T} u(s) \mathrm{d} s\right) z_{0}-z_{d}, \phi_{n}\right\rangle \phi_{n},
\end{aligned}
$$

and

$$
\begin{aligned}
& \left\|z_{u}(T)-z_{d}\right\|^{2} \\
& =\sum_{n=1}^{+\infty}\left\langle\exp \left(\lambda_{n} T\right) \exp \left(B \int_{0}^{T} u(s) \mathrm{d} s\right) z_{0}-z_{d}, \phi_{n}\right\rangle^{2} .
\end{aligned}
$$

If $u$ and $v$ are two distinct solutions to the problem (2), then (14) implies

$$
\int_{0}^{T} u(s) \mathrm{d} s=\int_{0}^{T} v(s) \mathrm{d} s .
$$

The control $w=(u+v) / 2$ lies in $U_{\text {ad }}(\Omega)$, i.e.,

$$
\begin{aligned}
z_{w}(T) & =S(T) \exp \left(B \int_{0}^{T} \frac{1}{2}[u(s)+v(s)] \mathrm{d} s\right) z_{0} \\
& =z_{u}(T)
\end{aligned}
$$

and

$$
\begin{aligned}
\|w\|_{L^{2}[0, T]}^{2} & =\frac{1}{4}\|u+v\|_{L^{2}[0, T]}^{2} \\
& <\frac{1}{2}\left[\|u\|_{L^{2}[0, T]}^{2}+\|v\|_{L^{2}[0, T]}^{2}\right] \\
& =\|u\|_{L^{2}[0, T]}^{2} .
\end{aligned}
$$

This contradiction implies that the minimum energy control is unique.

\section{Remark 3.}

1. The above results remain true in the case of multicontrols, i.e., when the system is described by

$$
\dot{z}(t)=A z(t)+\sum_{i=1}^{p} u_{i}(t) B_{i} z(t)
$$

where $\forall i, 1 \leq i \leq p, u_{i} \in L^{2}[0, T]$, and $B_{i}$ is a bounded linear operator on $Z$.

2. In the same way we can solve the following general problem:

$$
\left\{\begin{array}{l}
\min \|u\|_{L^{2}[0, T]}^{2} \\
\text { with } \\
\left\langle\chi_{\omega} z_{u}(T)-z_{d}(T), G\left(\chi_{\omega} z_{u}(T)-z_{d}(T)\right)\right\rangle_{L^{2}(\omega)} \\
+\int_{0}^{T}\left\langle\chi_{\omega} z_{u}(t)-z_{d}(t), Q\left(\chi_{\omega} z_{u}(t)-z_{d}(t)\right)\right\rangle \mathrm{d} t \\
\text { minimum, }
\end{array}\right.
$$

where $z_{d}$ is a desired regular function.

The problem associated with 15 is

$$
\left\{\begin{array}{l}
\min \Phi_{\epsilon}(u), \\
u \in L^{2}[0, T]
\end{array}\right.
$$

with

$$
\begin{aligned}
& \Phi_{\epsilon}(u) \\
& =\left\langle\chi_{\omega} z_{u}(T)-z_{d}(T), G\left(\chi_{\omega} z_{u}(T)-z_{d}(T)\right)\right\rangle_{L^{2}(\omega)} \\
& \quad+\int_{0}^{T}\left[\left\langle\left(\chi_{\omega} z_{u}(t)-z_{d}(t), Q\left(\chi_{\omega} z_{u}(t)-z_{d}(t)\right)\right\rangle_{L^{2}(\omega)}\right.\right. \\
& \left.\quad+\epsilon u^{2}(t)\right] \mathrm{d} t, \quad \epsilon>0
\end{aligned}
$$


whose solution is given by

$$
\begin{aligned}
u(t)= & -\frac{1}{\epsilon}\left\langle B z(t), P(t) z(t)-U^{*}(T, t) \chi_{\omega}^{*} G z_{d}(T)\right. \\
& \left.-\int_{t}^{T} U^{*}(s, t) \chi_{\omega}^{*} Q z_{d}(s) \mathrm{d} s\right\rangle .
\end{aligned}
$$

where $P$ is the self-adjoint and nonnegative operator solution of the equation

$$
\left\{\begin{array}{l}
\frac{\mathrm{d}}{\mathrm{d} t}\langle P(t) y, z\rangle+\langle P(t) y,(A+u(t) B) z\rangle \\
+\langle(A+u(t) B) y, P(t) z\rangle+\left\langle\chi_{\omega}^{*} Q \chi_{\omega} y, z\right\rangle=0, \\
P(T)=\chi_{\omega}^{*} G \chi_{\omega}, \text { where } y, z \in D(A) .
\end{array}\right.
$$

3. If $z_{d}(\cdot)$ is exactly reachable with the control $v$, then

$$
\begin{array}{cl}
u_{\epsilon} \rightarrow v & \text { in } L^{2}[0, T] \text { strongly, } \\
\chi_{\omega} z_{\epsilon} \rightarrow z_{d} & \text { in } C\left([0, T] ; L^{2}(\omega)\right) \text { strongly, }
\end{array}
$$

as $\epsilon \rightarrow 0$, where $u_{\epsilon}$ is a control which minimizes in $L^{2}[0, T]$ the quadratic cost

$$
\begin{aligned}
& J_{\epsilon}(u) \\
& \quad=\left\|\chi_{\omega} z_{u}(T)-z_{d}(T)\right\|_{L^{2}(\omega)}^{2} \\
& \quad+\int_{0}^{T}\left[\left\langle\chi_{\omega} z_{u}(t)-z_{d}(t), \chi_{\omega} z_{u}(t)-z_{d}(t)\right\rangle_{L^{2}(\omega)}\right. \\
& \left.\quad+\epsilon u^{2}(t)\right] \mathrm{d} t, \quad \epsilon>0 .
\end{aligned}
$$
set.

We now deal with the case where $U_{\text {ad }}(\omega)$ is an empty

Theorem 3. Suppose that $U_{\mathrm{ad}}(\omega)$ is empty. Then

$$
\lim _{\epsilon \rightarrow 0}\left\|\chi_{\omega} z_{\epsilon}(T)-z_{d}\right\|_{L^{2}(\omega)}^{2}=\inf _{z \in R(T)}\left\|\chi_{\omega} z-z_{d}\right\|_{L^{2}(\omega)}^{2} .
$$

Proof. Let

$$
F=\left\{\left\|\chi_{\omega} z-z_{d}\right\|_{L^{2}(\omega)} \mid z \in R(T)\right\} .
$$

Then, $F$ is a nonempty subset of $\mathbb{R}^{+}$. Therefore, $F$ has a lower bound denoted by $a$. According to Proposition 1, $\left(J_{\epsilon}\left(u_{\epsilon}\right)\right)_{\epsilon>0}$ is a decreasing sequence as $\epsilon \rightarrow 0$, and $J_{\epsilon}\left(u_{\epsilon}\right) \geq 0, \forall \epsilon>0$.

Hence, it converges in $\mathbb{R}$ towards a limit denoted by $J$. Similarly, $\left(\left\|\chi_{\omega} z_{\epsilon}(T)-z_{d}\right\|_{L^{2}(\omega)}\right)_{\epsilon>0}$ is a nonnegative and decreasing sequence. Thus, as $\epsilon \rightarrow 0$ it converges in $\mathbb{R}$ towards a limit denoted by $b$. Let us show that $b=a$. Suppose that $b>a$. Then there exists $v \in L^{2}[0, T]$ such that

$$
a<\left\|\chi_{\omega} z_{v}(T)-z_{d}\right\|_{L^{2}(\omega)}<b .
$$

Now,

$$
\begin{aligned}
& \left\|\chi_{\omega} z_{\epsilon}(T)-z_{d}\right\|_{L^{2}(\omega)}^{2}+\epsilon \int_{0}^{T} u_{\epsilon}^{2}(t) \mathrm{d} t \\
& \quad \leq\left\|\chi_{\omega} z_{v}(T)-z_{d}\right\|_{L^{2}(\omega)}^{2}+\epsilon \int_{0}^{T} v^{2}(t) \mathrm{d} t .
\end{aligned}
$$

Equations (17) and (18) imply that

$$
\int_{0}^{T} u_{\epsilon}^{2}(t) \mathrm{d} t \leq \int_{0}^{T} v^{2}(t) \mathrm{d} t
$$

Thus, according to Remark $2 U_{\text {ad }}^{T}(\omega)$ is nonempty, which is a contradiction.

\section{Remark 4.}

1. The family of controls $\left(u_{\epsilon}\right)_{\epsilon>0}$ is not bounded in $L^{2}[0, T]\left(\right.$ Remark 2) and for a fixed $\epsilon$ and for all $\chi_{\omega} z_{v}(T)$ such that

$$
\left\|\chi_{\omega} z_{\epsilon}(T)-z_{d}\right\|_{L^{2}(\omega)}=\left\|\chi_{\omega} z_{v}(T)-z_{d}\right\|_{L^{2}(\omega)}
$$

according to 18 we have

$$
\int_{0}^{T} u_{\epsilon}^{2}(t) \mathrm{d} t \leq \int_{0}^{T} v^{2}(t) \mathrm{d} t
$$

2. The approach used to solve the optimal control problem assumes a bounded control operator. However, the unbounded case may be carried out in a similar manner taking more regular controls which allow regular system states. This means that the control is selected such that the state $z$ is in $Z=L^{2}(\Omega)$.

\section{Numerical approach and simulations}

We have seen that, if an optimal control solution to the problem (2) exists, such a control may be approximated by the solution $u_{\epsilon}$ to the problem (3), which in turn may be implemented by the following formula:

$$
\left\{\begin{aligned}
u_{n+1}(t) & =-n\left\langle B z_{n}(t), P_{n}(t) z_{n}(t)-U_{n}^{*}(T, t) \chi_{\omega}^{*} z_{d}\right\rangle, \\
u_{0} & =0
\end{aligned}\right.
$$

where $P_{n}$ is the selfadjoint and nonnegative operator solution of the Riccati equation

$$
\left\{\begin{array}{l}
\frac{\mathrm{d}}{\mathrm{d} t}\left\langle P_{n}(t) y, z\right\rangle+\left\langle P_{n}(t) y,\left(A+u_{n}(t) B\right) z\right\rangle \\
+\left\langle\left(A+u_{n}(t) B\right) y, P_{n}(t) z\right\rangle=0 \\
P_{n}(T)=\chi_{\omega}^{*} \chi_{\omega} \text { with } y, z \in D(A)
\end{array}\right.
$$

whose solution can be achieved by the algorithm given by El Alami (1988). 
This allows us to consider the following algorithm:

Step 1: Initialize system data: $z_{0}, u_{0}=0$, desired state $z_{d}$, threshold accuracy $\varepsilon$, subregion $\omega$ and sensor location $b$.

Step 2: Until $\left\|u_{n+1}-u_{n}\right\| \leq \varepsilon$ repeat

$\odot$ Solve Eqn. (20) which gives $P_{n}$.

$\ominus$ Solve Eqn. (1) which gives $z_{n}(t)$.

$\ominus$ Compute $u_{n+1}$ by the formula (19).

The control $u_{n}$ steers the system to the desired state $z_{d}$ at time $T$.

To illustrate the above algorithm, consider the following examples.

Example 1. Let $\Omega=] 0,1[$ and consider the bilinear system described by the following evolution equation:

$$
\left\{\begin{aligned}
\frac{\partial z}{\partial t}(x, t)= & \alpha \frac{\partial^{2} z(x, t)}{\partial x^{2}}+\beta z(x, t) & & \\
& +\gamma u(t) z(x, t) & & \text { in } \Omega \times] 0, T[, \\
z(x, 0)= & z_{0}(x) & & \text { in } \Omega, \\
z(0, t)= & z(1, t)=0 & & \text { on }] 0, T[,
\end{aligned}\right.
$$

where $\alpha, \beta$ and $\gamma$ are positive real numbers. This equation may represent a simplified model of the temperature distribution in a furnace.

The system (21) looks like (1) with

$$
\tilde{A}=\alpha \frac{\partial^{2}}{\partial x^{2}}+\beta
$$

with the domain

$$
D(\tilde{A})=\left\{z \in H^{2}(0,1) \mid z(0)=z(1)=0\right\} .
$$

The operator $\tilde{A}$ admits a set of eigenfunctions $\phi_{i}(\cdot)$ associated with the eigenvalues $\lambda_{i}$ given by

$$
\phi_{i}(x)=\sqrt{2} \sin (i \pi x), \quad \lambda_{i}=\beta-\alpha i^{2} \pi^{2}, \quad i \geq 1 .
$$

The solution (21) is approximated by

$$
z(x, t) \simeq \sum_{i=1}^{M} a_{i}(t) \phi_{i}(x) .
$$

Let $z_{0}(x)=\sin (\pi x), z_{d}(x)=8 x(1-x), \alpha=$ $0.01, \beta=0.01, \gamma=0.02, \varepsilon=0.0001$ and $T=1$. Augmenting the truncation order $M$ beyond 5 does not improve the simulation results.

Using the above algorithm for different regions of $\omega$ and after the 7-th iteration, we have

(i) Case of $\omega=] 0.4,0.6[$ : see Figs. 1 and 2,

(ii) Case of $\omega=] 0.6,1[$ : see Figs. 3 and 4.

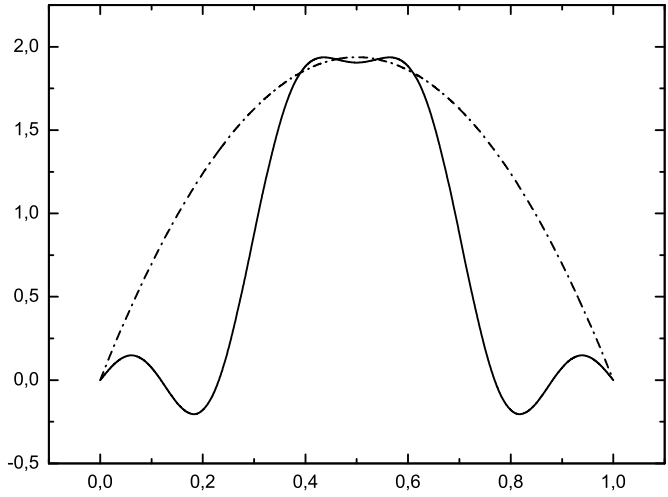

Fig. 1. Desired state $z_{d}$ (dashed line) and the reached state $z_{u_{7}}(T)$ (continuous line) in $\omega$.

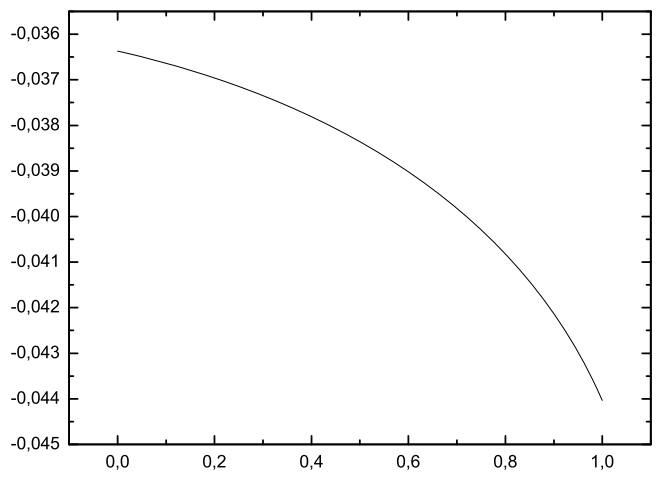

Fig. 2. Optimal control function $u^{\star}(\cdot) \simeq u_{7}(\cdot)$.

(iii) Case of $\omega=] 0.8,1[$ : see Figs. 5 and 6.

Example 2. Let us consider the bilinear system with the domain $\Omega=] 0,1$ [ described by the following equation:

$$
\left\{\begin{aligned}
\frac{\partial z}{\partial t}(x, t) & =\alpha \frac{\partial^{2} z(x, t)}{\partial x^{2}}+\beta z(x, t)+ & & \tilde{\gamma} u(t) z(x, t) \\
& +\delta(x-b) u(t) & & \text { in } \Omega \times] 0, T[, \\
z(x, 0) & =z_{0}(x) & & \text { in } \Omega, \\
z(0, t) & =z(1, t)=0 & & \text { on }] 0, T[.
\end{aligned}\right.
$$

The operator

$$
\tilde{A}=\alpha \frac{\partial^{2}}{\partial x^{2}}+\beta
$$

has the domain $D(\tilde{A})=\left\{z \in H^{2}(0,1) \mid z(0)=z(1)=\right.$ $0\}$ and $\delta$ is the Dirac delta. Let $z_{0}(x)=6.4 x(1-x), \alpha=$ $0.01, \beta=0.01, \tilde{\gamma}=0.02, \varepsilon=10^{-4}, T=1$ and $b=0.1$.

For

$$
\omega=[0.35,0.65]
$$




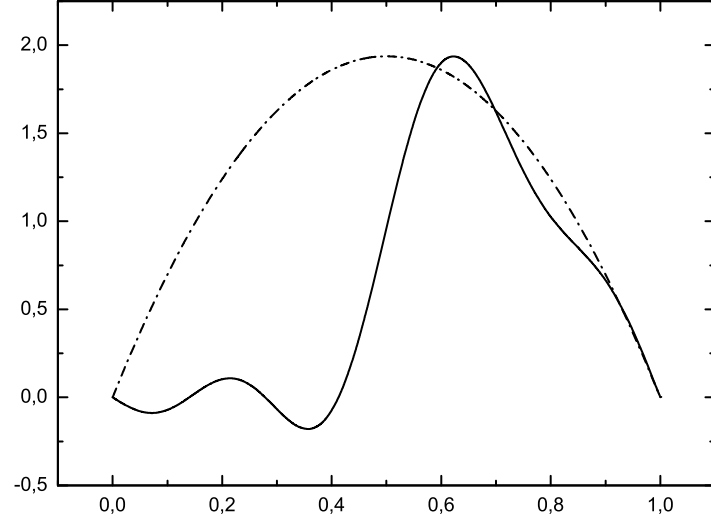

Fig. 3. Desired state $z_{d}$ (dashed line) and the reached state $z_{u_{7}}(T)$ (continuous line) in $\omega$.

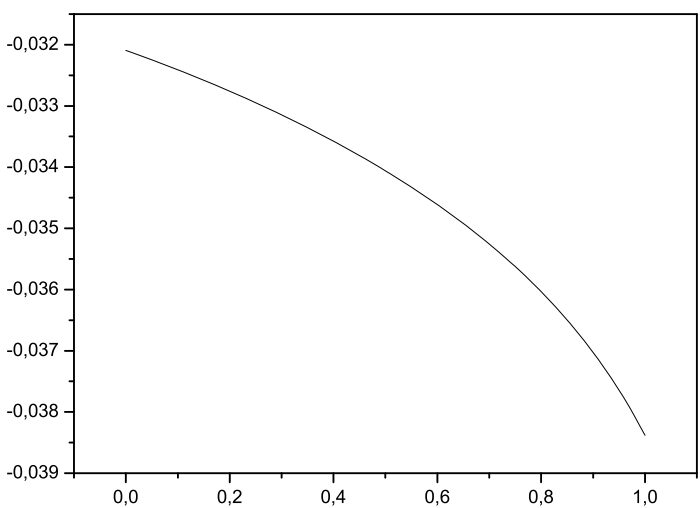

Fig. 4. Optimal control function $u^{\star}(\cdot) \simeq u_{7}(\cdot)$.

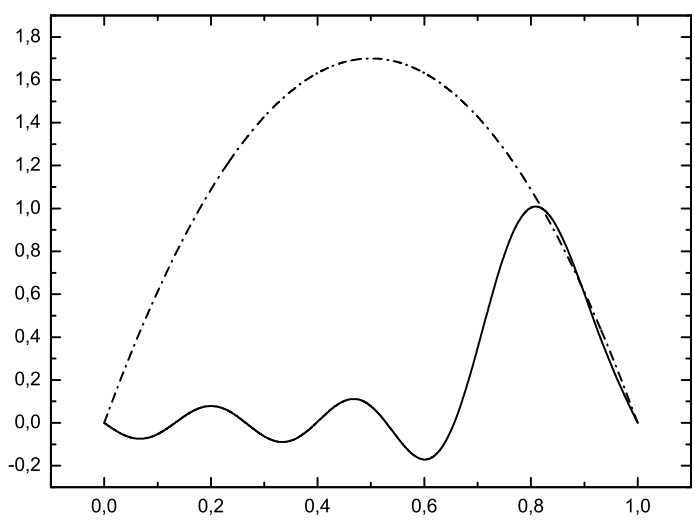

Fig. 5. Desired state $z_{d}$ (dashed line) and the reached state $z_{u_{7}}(T)$ (continuous line) in $\omega$.

and

$$
z_{d}=\left\{\begin{array}{cl}
1.5+300(x-0.3)^{2} & \\
\times(x-0.7)^{2} & \text { if } x \in[0.35,0.65], \\
0 & \text { otherwise }
\end{array}\right.
$$

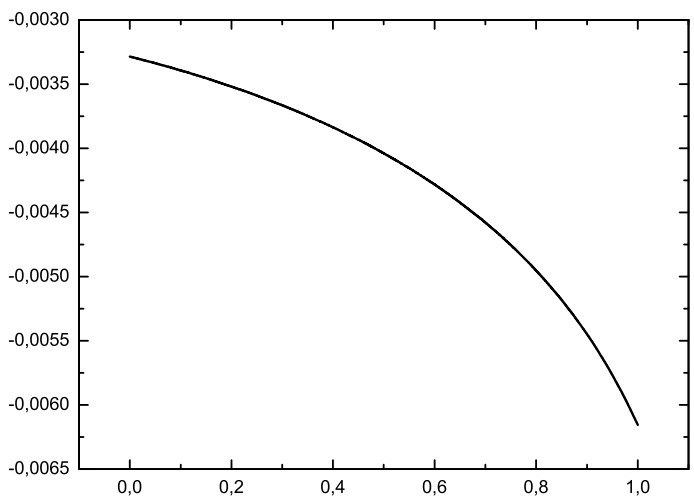

Fig. 6. Optimal control function $u^{\star}(.) \simeq u_{7}($.$) .$

application of the above algorithm gives the results presented in Figs. 7 and 8.

\section{Conclusion}

A regional controllability problem for bilinear systems was considered and an optimal control was characterized. Under adding conditions, the uniqueness of such a control was proved. Moreover, a numerical approach was developed based on a quadratic control problem. The obtained results were successfully tested through numerical examples and simulations. Many questions remain still open, e.g., the extension of the present results to a boundary subregion. The case of systems with time delays would also be very interesting.

\section{Acknowledgment}

This work was carried out with the help of the Hassan II Academy of Sciences and Technologies.

\section{References}

Ball, J.M., Marsden, J.E. and Slemrod, M. (1982). Controllability for distributed bilinear systems, SIAM Journal on Control and Optimization 20(4): 575-597.

Bradley, M.E. and Lenhart, S. (2001). Bilinear spatial control of the velocity term in a Kirchhoff plate equation, Electronic Journal of Differental Equations (27): 1-15.

El Alami, N. (1988). Algorithms for implementation of optimal control with quadratic criterion of bilinear systems, in A. Bensoussan and J.L. Lions (Eds.) Analysis and Optimization of Systems, Lecture Notes in Control and Information Sciences, Vol. 111, Springer-Verlag, London, pp. 432-444, (in French).

El Jai, A., Simon, M.C., Zerrik, E. and Prirchard, A.J. (1995). Regional controllability of distributed parameter systems, International Journal of Control 62(6): 1351-1365. 


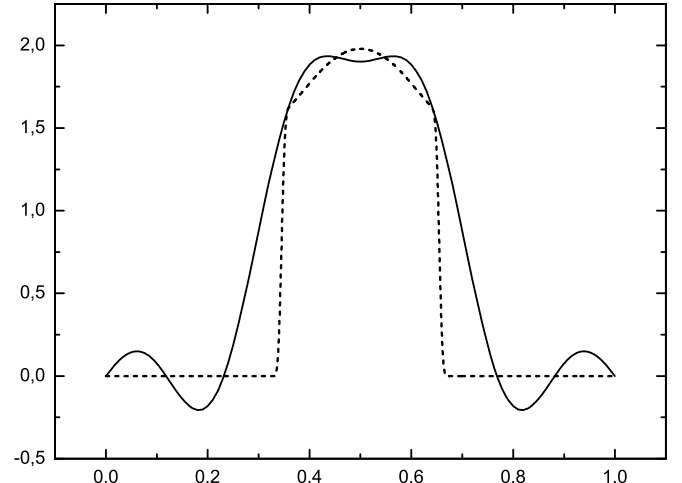

Fig. 7. Desired state $z_{d}$ (dashed line) and the reached state $z_{u_{6}}(T)$ (continuous line) in $\omega$.

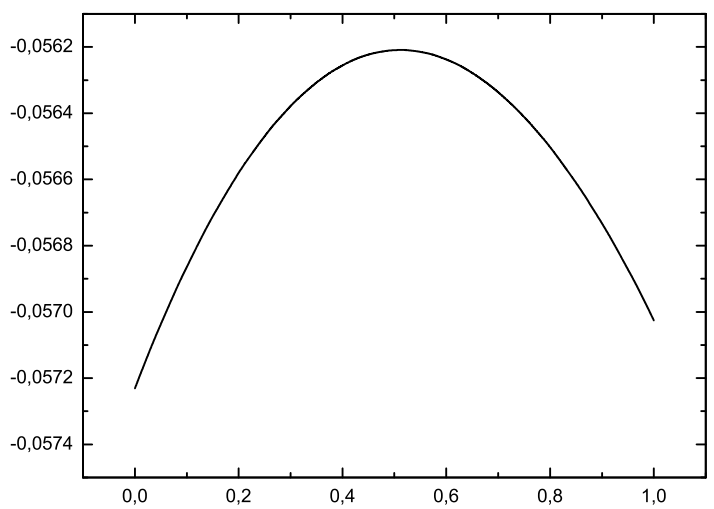

Fig. 8. Optimal control function $u^{\star}(\cdot) \simeq u_{6}(\cdot)$.

Joshi, H.R. (2005). Optimal control of the convective velocity coefficient in a parabolic problem, Nonlinear Analysis $\mathbf{6 3}$ (5-7): 1383-1390.

Kato, T. (1995). Perturbation Theory for Linear Operators, Springer Verlag, Berlin/Heidelberg.

Khapalov, A.Y. (2002a). Global non-negative controllability of the semilinear parabolic equation governed by bilinear control, ESAIM: Control, Optimisation and Calculus of Variations 7: 269-283.

Khapalov, A.Y. (2002b) On bilinear controllability of the parabolic equation with the reaction-diffusion term satisfying Newton's, Journal of Computational and Applied Mathematics 21: 1-23.

Khapalov, A.Y. (2010). Controllability of Partial Differential Equations Governed by Multiplicative Controls, Lecture Notes in Mathematics, Vol. 1995, Springer, Berlin, p. 284.

Lenhart, S. and Liang, M. (2000). Bilinear optimal control for a wave equation with viscous damping, Houston Journal of Mathematics 26(3): 575-595.
Pazy, A. (1983). Semigroups of Linear Operators and Applications to Partial Differential Equations, Springer-Verlag, New York, NY.

Zerrik, E., Ouzahra, M. and Ztot, K. (2004). Regional stabilization for infinite bilinear systems, IEE: Control Theory and Applications 151(1): 109-116.

Zerrik, E. and Kamal, A. (2007). Output controllability for semi linear distributed parabolic system, Journal of Dynamical and Control Systems 13(2): 289-306.

Zerrik, E., Larhrissi, R. and Bourray, H. (2007). An output controllability problem for semi linear distributed hyperbolic system, International Journal of Applied Mathematics and Computer Science 17(4): 437-448, DOI: 10.2478/v10006007-0035-y.

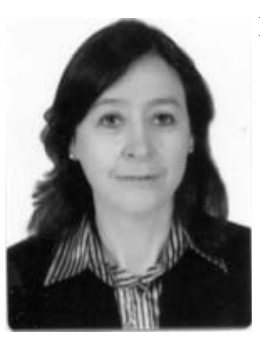

Karina Ztot is a professor at the Moulay Ismail University of Meknes in Morocco. She obtained her doctorate d'État in bilinear systems control and analysis (1996) at the Mohammadia Graduate School of Engineering of Rabat, Morocco. Now she is a member of the MACS (Modelling Analysis and Control of Systems) research team at the Moulay Ismail University of Meknes.

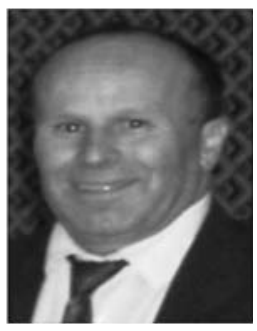

El Hassan Zerrik is a professor at the Moulay Ismail University of Meknes in Morocco. He has been an assistant professor in the Faculty of Sciences of Meknes and a researcher at the University of Perpignan, France. He obtained his doctorate d'État in systems regional analysis (1993) at the Mohammed V University of Rabat, Morocco. Professor Zerrik has authored many papers in the area of systems analysis and control. Now he is the head of the MACS (Modelling Analysis and Control of Systems) research team at the Moulay Ismail University of Meknes.

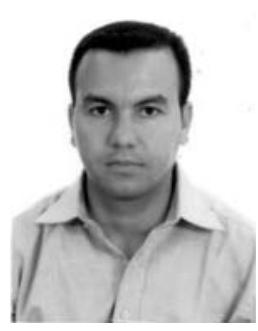

Hamid Bourray is a professor at the Moulay Ismail University of Meknes in Morocco and an assistant professor in the Faculty of Multidisciplinary Research of Errachidia, Morocco. He obtained his doctorate in systems analysis (2002) at the Moulay Ismail University of Meknes. Professor Bourray has authored many papers in the area of systems analysis and control. He is a researcher at the TICOS team at the Moulay Ismail University of Meknes.

Received: 2 May 2010

Revised: 23 November 2010

Re-revised: 20 January 2011 